\title{
Association Between State-Level Income Inequality and COVID-19 Cases and Mortality in the USA
}

J Gen Intern Med 35(9):2791-3

DOI: $10.1007 / \mathrm{s} 11606-020-05971-3$

(C) Society of General Internal Medicine 2020

\section{INTRODUCTION}

COVID-19, caused by the novel coronavirus SARS-CoV-2, has resulted in the largest pandemic in 100 years. The USA has been particularly impacted, reporting a third of the cases and a quarter of the deaths worldwide. In recent weeks, the unequal impact of COVID-19 across communities has become glaringly apparent. Data from New York and Chicago indicate that African American and Hispanic people experience disproportionately higher rates of COVID-19 infection and mortality. ${ }^{1,2}$ Inequality may compound these disparities further through economic segregation, decreased social mobility, and lower access to medical care. ${ }^{3}$ Given that low-income individuals are more likely to be in essential occupations with a high exposure risk and have less access to healthcare, income inequality may exacerbate the impact of the COVID-19 outbreak.

\section{METHODS}

We examined the association between income inequality and the number of COVID-19 cases and deaths. State income inequality data - as measured by the Gini index-were extracted from the 2018 American Community Survey. The number of cases and deaths was calculated using the COVID-19 Dashboard, a data set hosted by the Center for Systems Science and Engineering at Johns Hopkins University. ${ }^{4,5}$ We limited our analysis to the 50 states from January 22 , 2020, through April 13, 2020.

First, we performed simple correlation analyses between the state-level Gini index and the number of cases and deaths per 100,000 population due to COVID-19 using the Spearman rank-order correlation test. To account for the right-skewed distribution, we log-transformed the data on the number of COVID-19 cases and deaths.

Second, using multivariable regressions, we examined the associations between the state-level Gini index and logtransformed number of cases and deaths due to COVID-19 adjusting for potential confounders. The adjustment variables

Received May 7, 2020

Accepted June 5, 2020

Published online June 24, 2020 included the proportion of the population $65+$ years, female, African American, Hispanic, and below poverty; median household income; the number of tests performed per capita; doctors per capita (2018-2019 Area Health Resource File); beds per capita (2009-2018 American Hospital Association Annual Survey); and whether a state had a stay-at-home or shelter-in-place policy (no order, order in some parts of the state, statewide order; the New York Times database). ${ }^{6}$

This study was exempted from review by the institutional review board of UCLA.

\section{RESULTS}

On April 13,2020, there were a total of 577,414 cases and 23,424 deaths across 50 states. The number of cases ranged from 28.7 to $1,006.2$ cases per 100,000 (median. 73.0; IQR, 47.7-133.6). The mortality rates ranged from 0.17 to 51.7 deaths per 100,000 (median, 2.0; IQR, 1.2-4.5).

We observed positive correlations between the Gini index and the number of cases (correlation coefficient $=0.38 ; P=$ 0.006 ) and deaths (correlation coefficient $=0.44 ; P=0.002$ ) due to COVID-19 (Fig. 1). After adjusting for potential confounders, we found that states with a higher Gini index experienced a larger number of deaths due to COVID-19 (adjusted percent change for one unit increase in Gini index, $+27.2 \%$; $95 \% \mathrm{CI},+3.5 \%$ to $+56.3 \% ; P=0.02$ ); the Gini index was marginally associated with the number of COVID-19 cases $(+13.5 \% ; 95 \% \mathrm{CI},+0.0 \%$ to $+30.0 \% ; P=0.07)($ Table 1$)$.

\section{DISCUSSION}

We found that states with higher income inequality experienced a higher number of deaths due to COVID-19. These findings suggest that social factors such as income inequality may explain why some parts of the USA are hit harder by the COVID-19 pandemic than others.

Our study has limitations. First, as is the case with observational studies, there is a possibility of residual confounding, including from comorbidities. However, we included the proportion of the population $65+$ years which may be a proxy of underlying health risks of the populations. Second, the use of state-level data precluded us from making any inferences about individual-level associations between inequality and COVID-19 infections. 
(a) The number of COVID-19 cases

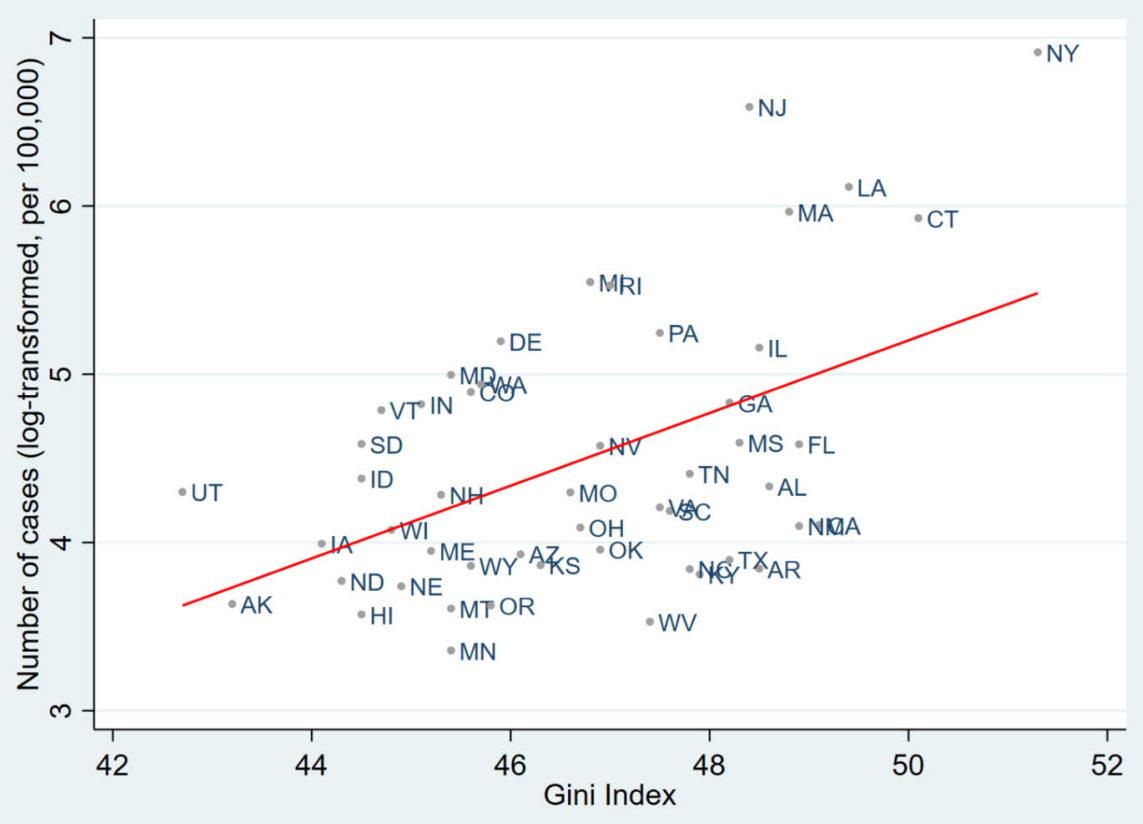

Correlation coefficient $=0.38 ; \mathrm{P}=0.006$

\section{(b) The number of COVID-19 deaths}

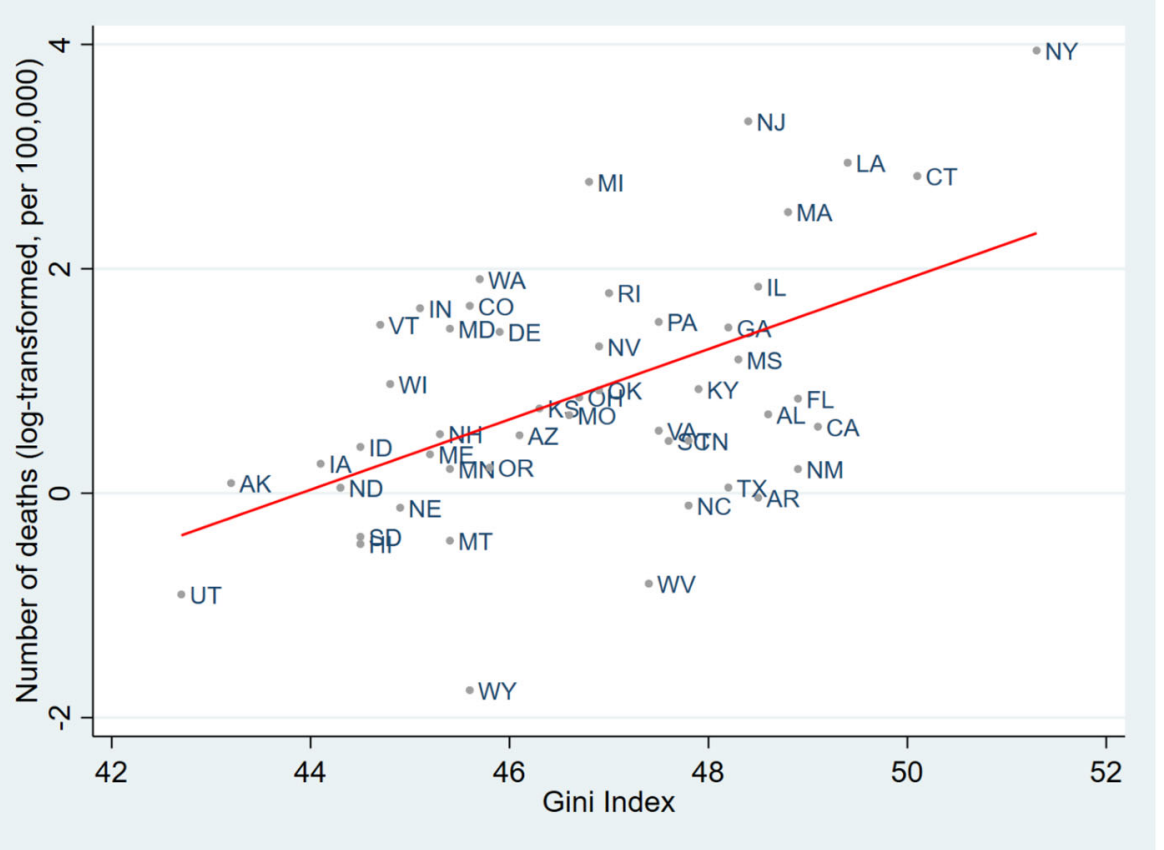

Correlation coefficient $=0.44 ; P=0.002$

Figure 1 The unadjusted correlation between the state-level Gini index and the number of COVID-19 cases (a) and deaths (b). 
Table 1 The Adjusted Association Between the State-Level Gini Index and the Number of COVID-19 Cases and Deaths

\begin{tabular}{lll}
\hline \hline & $\begin{array}{l}\text { Change in the number of COVID-19 } \\
\text { cases/deaths }(\mathbf{9 5 \%} \text { CI) }\end{array}$ & $\begin{array}{l}\boldsymbol{P} \\
\text { value }\end{array}$ \\
\hline $\begin{array}{l}\text { Cases per } \\
100,000\end{array}$ & $+13.5 \%(0.0 \%$ to $+30.0 \%)$ & 0.07 \\
$\begin{array}{l}\text { Deaths per } \\
100,000\end{array}$ & $+27.2 \%(+3.5 \%$ to $+56.3 \%)$ & 0.02 \\
\hline
\end{tabular}

*Adjusted for the following state-level variables: proportion of the population over 65 years, female, African American, Hispanic, and below poverty; median household income; the number of tests performed per capita; total doctors per capita; total beds per capita; and whether a state had a stay-at-home or shelter-in-place policy on April 1, 2020

Our findings should be informative for policymakers considering additional policies to mitigate the effects of COVID19 on the most financially vulnerable.

Carlos Irwin Oronce, $M D, M P H^{1,2}$

Christopher A. Scannell, $\mathrm{MD}, \mathrm{PhD}^{1,2}$

Ichiro Kawachi, $\mathrm{MD}, \mathrm{Ph} \mathrm{D}^{3}$

Yusuke Tsugawa, $\mathrm{MD}, \mathrm{Ph} \mathrm{D}^{2,4}$

${ }^{1}$ VA Greater Los Angeles Healthcare System and the National Clinician Scholars Program,

Los Angeles, CA, USA

${ }^{2}$ Division of General Internal Medicine and Health Services Research, David Geffen School of Medicine at UCLA,

1100 Glendon Avenue Suite 850, Los Angeles, CA 90024, USA

${ }^{3}$ Department of Social and Behavioral Sciences, Harvard T.H. Chan School of Public Health,

Boston, MA, USA

${ }^{4}$ Department of Health Policy and Management, UCLA Fielding School of Public Health,

Los Angeles, CA, USA
Corresponding Author: Carlos Irwin A. Oronce, MD, MPH; Division of General Internal Medicine and Health Services Research, David Geffen School of Medicine at UCLA, 1100 Glendon Avenue Suite 850, Los Angeles, CA 90024, USA (e-mail: coronce@mednet.ucla.edu).

Funding Information Drs. Oronce and Scannell are supported by the National Clinician Scholars Program at the University of California, Los Angeles, and by the VA Office of Academic Affiliations through the VA/National Clinician Scholars Program.

\section{Compliance with Ethical Standards:}

Conflict of Interest: The authors declare that they do not have a conflict of interest.

Disclaimer: The contents do not represent the views of the U.S. Department of Veterans Affairs or the US Government.

\section{REFERENCES}

1. Elignon J, Burch ADS, Searcey D, et al. Black Americans Face Alarming Rates of Coronavirus Infection in Some States. 7 April 2020. Accessed at https://www.nytimes.com/2020/04/07/us/coronavirus-race.html on 19 April 2020.

2. Thebault R, Tran AB, Williams V. African Americans are at higher risk of death from coronavirus. 7 April 2020. Accessed at https://www. washingtonpost.com/nation/2020/04/07/coronavirus-is-infecting-killing-black-americans-an-alarmingly-high-rate-post-analysis-shows/? arc404=true on 19 April 2020.

3. Bor J, Cohen GH, Galea S. Population health in an era of rising income inequality: USA, 1980-2015. Lancet. 2017;389(10077):1475-1490.

4. Dong E, Du H, Gardner L. An interactive web-based dashboard to track COVID-19 in real time. Lancet Infect Dis. 2020;20(5):533-534.

5. Johns Hopkins University Center for Systems Science and Engineering. Novel Coronavirus (COVID-19) Cases. Accessed at https://github.com/ CSSEGISandData/COVID-19/tree/master/csse_covid_19_data/csse_ covid_19_daily_reports on 13 April 2020.

6. Mervosh S, Lu D, Swales V. See Which States and Cities Have Told Residents to Stay at Home. 20 April 2020. Accessed at https://www. nytimes.com/interactive/2020/us/coronavirus-stay-at-home-order.html on 22 April 2020.

Publisher's Note: Springer Nature remains neutral with regard to jurisdictional claims in published maps and institutional affiliations. 